Recepción: 24/ 07 / 2018

Aceptación: 16 / 09 / 2018

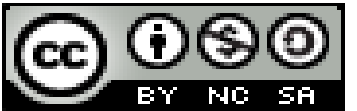

Ciencias económicas y empresariales

Publicación: 01 / 11 / 2018

Artículo de investigación

\title{
La crisis financiera global y su efecto en la economía del Ecuador
}

The global financial crisis and its effect on the economy of Ecuador A crise financeira global e seus efeitos na economia do Equador

\author{
Ángel C. Alvarado-Gastiaburo I \\ angel.alvaradog@ug.edu.ec \\ Nelly S. Vergara-Díaz II \\ nelly.vergaradi@ug.edu.ec
}

\section{Correspondencia: angel.alvaradog@ug.edu.ec}

\footnotetext{
I Magister en Negocios Internacionales y Gestión de Comercio Exterior, Licenciado en Ciencias Sociales y Políticas, Abogado de los Tribunales y Juzgados de la República del Ecuador, Economista, Docente de la Universidad de Guayaquil, Guayaquil, Ecuador.

II Docente de la Universidad de Guayaquil, Guayaquil, Ecuador.
} 


\title{
Resumen
}

Se presenta una investigación documental, mediante una amplia revisión bibliográfica, estudio de casos, revisión de artículos relacionados a la crisis financiera y económica mundial y sus efectos en el sistema bancario de Ecuador, con un enfoque cualitativo, con el objetivo de comprender las características del sistema financiero en este país, considerando asimismo, que presenta un sistema poco sofisticado y pequeño, que permanece fragmentado, donde está presente la existencia del control gubernamental sobre varias de las principales instituciones financieras, a pesar de la promoción al acceso a los servicios y la democratización del crédito, para así poder aumentar la productividad y la competitividad de sectores específicos de acuerdo a los lineamientos del Plan Nacional de Desarrollo. Se concluye, que los cambios que ha venido sufriendo Ecuador, en base al control de su Sistema Financiero es notable, entendiendo que estabilizarse, tomará algunos años más, requerirá un alto financiamiento y demandará, sobre todo, un real cambio de orientación del modelo económico.

Palabras clave: crisis financiera; sistema financiero; instituciones financieras; Ecuador.

\begin{abstract}
A documentary research is presented, through an extensive literature review, case study, review of articles related to the global financial and economic crisis and its effects on the banking system of Ecuador, with a qualitative approach, with the aim of understanding the characteristics of the financial system in this country, also considering that it presents an unsophisticated and small system, which remains fragmented, where the existence of government control over several of the main financial institutions is present, despite the promotion of access to services and democratization of credit, in order to increase productivity and competitiveness of specific sectors according to the guidelines of the National Development Plan. It is concluded that the changes that Ecuador has been experiencing, based on the control of its Financial System is remarkable, understanding that stabilizing, will take a few more years, will require a high financing and will demand, above all, a real change of orientation of the economic model.
\end{abstract}

Key words: financial crisis; finance system; financial institutions; Ecuador. 


\section{Resumo}

Pesquisa documental é apresentada através de uma extensa revisão da literatura, estudos de caso, revisão relacionada com a crise económica e financeira mundial e seus efeitos sobre o sistema bancário no Equador, com abordagem qualitativa, a fim de compreender as características de itens sistema financeiro neste país, considerando também apresenta um sistema sofisticado e pequenos, que permanece fragmentado, onde a existência de controle do governo sobre várias grandes instituições financeiras, apesar de promover o acesso aos serviços está presente e democratização do crédito, a fim de aumentar a produtividade e a competitividade de setores específicos de acordo com as diretrizes do Plano Nacional de Desenvolvimento. Conclui-se que as mudanças têm sofrido Equador, com base no controle de seu sistema financeiro é notável, entendimento de que estabilizar, levar alguns anos, requerem alta financiamento e exigirá, acima de tudo, uma verdadeira mudança de direção do modelo econômico.

Palavras chave: crise financeira; sistema financeiro; instituições financeiras; Equador.

\section{Introducción}

La crisis financiera mundial, fue transmitida al mundo en desarrollo a través del agudo cambio negativo de los impactos positivos que se experimentaron durante el período del auge de 20032007. Si bien el impacto financiero afectó más a los países de ingresos medios, el colapso del comercio afectó a todas las economías; su fortaleza entonces, dependió más de la composición de las materias primas para las exportaciones y la apertura al comercio que del grado de desarrollo. Se identificó, además, un tercer canal de transmisión: la caída de las remesas de los trabajadores y trabajadoras migrantes, que tuvo un impacto significativo en su subgrupo de países en desarrollo, sobre todo en las economías pequeñas (Libertad Digital Economía, s.f.), extendiéndose al resto del mundo por el fenómeno de la globalización de los flujos de capitales.

Al mismo tiempo, a raíz de la crisis financiera, se empieza a hablar de una crisis económica que afectaría no sólo al aspecto financiero de la economía, sino también, a otros factores que se producen en el año 2008, tales como: el aumento de los precios de las materias primas, con lo que podríamos hablar de una crisis alimentaria y de una crisis de confianza de los mercados. La relación entre recesión y crisis económica es por lo tanto evidente y muchas veces, se utiliza 
indistintamente el término crisis económica cuando se da un crecimiento del PNB. negativo durante dos trimestres consecutivos (Peláez, 2011).

\section{Planteamiento del Problema}

El sistema financiero ha mejorado la administración de la liquidez con sistemas «más técnicos de control y un mejor manejo de concentración de depósitos», que se suman a mejor tecnología y estadísticas. Los niveles de solvencia se han mantenido estables, gracias a la capitalización de utilidades que han sido impuestas, en algunos casos, por los organismos de control.

No obstante, a pesar del fortalecimiento del sistema, la calificadora de riesgos señala que hay un alto nivel de asimetría entre las instituciones más grandes y pequeñas, en cuanto a fortalezas y debilidades. A esto se suma, que la estructura económica del país, reducida en ingresos y con poca flexibilidad de fondeo, limita las calificaciones del sistema financiero ecuatoriano, como consecuencia de la dependencia de Ecuador, de las materias primas y la ausencia de un fondo de estabilización para épocas de crisis, lo que generaría incertidumbre en la liquidez de la economía e «influye directamente en el desarrollo del sistema financiero» (Aguilera, 2015).

\section{Metodología}

Se fundamenta en una investigación documental, mediante una amplia revisión bibliográfica, estudio de casos, revisión de artículos relacionados a la crisis financiera y económica mundial y sus efectos en el sistema bancario de Ecuador, con un enfoque cualitativo frente al objeto de estudio.

\section{Desarrollo}

La crisis financiera mundial se origina en Estados Unidos en el año de 1929, tras la 'gran depresión', la cual generó el hundimiento de la bolsa de Nueva York, así como la quiebra de grandes bancos, la paralización de negocios y mala política económica en Estados Unidos.

La 'gran depresión' también conocida como crisis del 29, ocasionó una crisis mundial que se alargó durante la década de los 30, antes de la Segunda Guerra Mundial y terminó en diferentes momentos de estos años y principios de los 40, según el país. Fue la mayor y más importante depresión económica de la historia moderna y se utiliza en el siglo 21 como punto de referencia, sobre lo que podría ser una futura caída de la economía mundial (Pérez, s.f.). 
Esta se originó, por la causa de algunos factores, siendo uno de éstos, la quiebra masiva de bancos, dejando sin ahorro a los depositantes. Los depósitos de dinero no contaban con seguridad, esto quiere decir que, si el banco quebraba, los ahorradores se quedaban sin dinero y esto fue lo que ocurrió. Al ver esta situación, los ahorradores de otros bancos los cuales eran solventes, empezaron hacer retiros masivos de sus ahorros, sin embargo, a pesar de su solvencia, no pudieron hacer frente a estos gigantescos retiros por lo que también quebraron.

La paralización de los negocios fue otro de los factores que afectó este proceso de crisis ya que, con la quiebra de bancos, los negocios tuvieron difícil acceso a créditos para financiar sus empresas. Las empresas trataron de mantenerse recortando gastos, entre ellos los sueldos de los trabajadores, por lo que se produjo la época de despidos masivos.

La mala política económica del gobierno también influyó en esta crisis, estableciendo tarifas a las importaciones; la teoría de esta política era proteger la producción interna, pero los países europeos, que fueron los mayores perjudicados con esta medida, decidieron tomar políticas similares en venganza. Y así, Estados Unidos se quedó sin mercado internacional, lo que agravó la crisis interna.

En 1944, tras la II Guerra Mundial se crean los acuerdos de Bretton Woods (EEUU), para las relaciones comerciales y financieras entre los países más industrializados del mundo; también se crea el Banco Mundial (BM) y el Fondo Monetario Internacional (FMI) y se extiende el uso del dólar, como moneda referencial internacional (Libertad Digital Economía, s.f.).

Para el año 1971, se suspende la convertibilidad con el oro y la moneda desciende su valor en un $10 \%$. Dos años después, se volvió a devaluar la moneda, con lo que acabó finalmente con el patrón oro. Así comenzó la época de los cambios flotantes, en función de la evolución de los mercados de capital (Libertad Digital Economía, s.f.).

Durante el año 1973, tras el corte de suministro de los países OPEP, en la primera crisis del petróleo, se incrementa el precio del crudo de 2,50 a 11,50 dólares en 1974. Esto elevó la factura energética de Occidente y provocó una fuerte crisis en los países más industrializados (Libertad Digital Economía, s.f.). 
El 19 de octubre de 1987, el lunes Negro, millones de inversores se lanzaron en masa a vender sus acciones en la Bolsa de Nueva York, debido a la creencia generalizada del manejo inapropiado de la información confidencial y la adquisición de empresas con dinero procedente de créditos. Ese día, el índice Dow Jones de Industriales se desplomó 508 puntos $(-22,6 \%)$ y arrastró a las bolsas europeas y japonesas. Se intensifica la coordinación en la política monetaria internacional y en los principales asuntos económicos (Libertad Digital Economía, s.f.),

Para el año 2007, Estados Unidos, tras la crisis financiera que se originó en 1930, en consecuencia, en una relajación en la evaluación de riesgo, sufre su mayor crisis siendo el detonante, el estallido de una enorme burbuja inmobiliaria, que reveló que los bancos habían extendido hipotecas basura (subprime) a personas que no podían pagarlas, con la expectativa de que el precio de las viviendas seguiría subiendo. Esas hipotecas fueron titulizadas y vendidas en los mercados, lo que causó cientos de miles de millones de dólares en pérdidas a los inversores (Libertad Digital Economía, s.f.).

En el año 2008, se inició una fase más profunda de la crisis, tras la intervención del Gobierno en los bancos hipotecarios más grandes de Estados Unidos, con inyecciones de más de US \$ 200 mil millones. Las consecuencias de la caída del cuarto banco de inversión más importante de Wall Street, transmitieron el mensaje a los mercados de que no se sabía qué firmas serían capaces de pagar sus deudas, estallando el pánico entre financieras e inversionistas de todo el mundo. Lo que había empezado como una crisis de liquidez, se había transformado en una crisis de insolvencia (Libertad Digital Economía, s.f.).

Este desplome, produjo una contracción de la explotación de productos industrializados. El Producto Interno Bruto (PIB.) del 6\%, a finales de 2008, pasó al 4\% en el primer trimestre de 2009, sobre todo en China e India, que ralentizaron su producción. La recuperación vino en el tercer trimestre de 2009 y Asia se situó por encima de los niveles pre-crisis, mientras otras regiones se mantuvieron debajo, particularmente Europa Central y del Este (Libertad Digital Economía, s.f.). 
Este proceso afectó de una forma negativa a la producción mundial, aumentando el desempleo y sub-empleo y produjo un importante proceso de ajustes a la baja en los valores de los stocks reales y financieros, creando consecuencias como:

La recuperación económica se torna lenta y gradual.

Realización de cambios en los escenarios financieros y/o profundización de regulaciones prudenciales, aspecto que, en corto plazo, implican niveles de reservas bancarias más elevadas y acotamiento en la liquidez, con el fin de evitar excesivos apalancamientos y control sobre la generación de nuevos instrumentos financieros.

En 1994, el gobierno de México es incapaz de mantener su tipo de cambio fijo frente al dólar y anuncia la devaluación de la moneda. La falta de confianza en su economía, provoca una gran salida de capital, los créditos se interrumpen, la producción disminuye y el desempleo incrementa más de un 60 por ciento. Sus efectos negativos sobre el resto de América Latina, se bautizaron como el "Efecto Tequila".

En 1997, brota la crisis de Asia. En julio de este año, se devaluó la moneda tailandesa y tras ella cayeron las de Malasia, Indonesia y Filipinas, lo que repercutió también en Taiwán, Hong Kong y Corea del Sur. Arrastró al resto de economías y esta crisis, que en un primer momento parecía regional, acabó convirtiéndose en la primera crisis global. El FMI, elaboró una serie de paquetes de rescate para salvar a las economías más afectadas y promovió reformas estructurales.

Para el año de 1998, Rusia colapsa su sistema Bancario Nacional, con una suspensión parcial de pagos internacionales, la devaluación de su moneda y la congelación de los depósitos en divisas. El FMI, concedió créditos multimillonarios para atajar la caída libre de su divisa y que el impacto fuera irreparable en el mercado internacional. También instó a sus autoridades, a acelerar las reformas estructurales internas para fortalecer su sistema financiero.

Entre los años 2001-2002, el gobierno de Argentina, carece de fondos para mantener la paridad fija del peso con el dólar e impone restricciones a la retirada de depósitos bancarios (corralito), para evitar la fuga de capitales. En diciembre de 2001, suspende el pago de la deuda, de casi 100.000 millones de dólares, lo que constituye la mayor quiebra de la historia. En enero de 2002, 
el presidente Eduardo Duhalde, se ve obligado a terminar con la paridad y convierte en pesos los depósitos bancarios en dólares.

La crisis de la deuda en Europa, se da entre los años 2009-2010. El nuevo gobierno de Grecia, reconoce que el déficit del país es mucho superior a lo revelado anteriormente, lo que hace que el interés de sus bonos se dispare en los mercados. La Unión Europea y el FMI, negocian durante meses un programa de ayuda para evitar una extensión de la crisis a otras economías con problemas similares, en particular Portugal, España, Irlanda e Italia, aunque los temores en los mercados hunden el valor del Euro.

La crisis mundial tuvo repercusiones particulares en el caso del Ecuador, dadas las limitaciones de su economía dolarizada. Cuando la crisis se transmitió, el país no tuvo acceso a medidas de política monetaria y se vio privado de varios instrumentos para enfrentarla. En consecuencia, se recurrió a medidas de restricción de importaciones.

El estudio de la CEPAL (2008), en Panorama Social de América Latina, muestra los siguientes resultados para el año 2006, justo antes de la llegada de Rafael Correa a la Presidencia de la República y antes que se viniera la crisis económica internacional:

El índice de Gini, para Ecuador en el año 2006 fue de 0,507;

El 19,3\% de la población poseía un ingreso inferior al 50\% de la mediana;

El 10\% de la población más pobre, solo disponía del 1,2\% del total de ingresos;

El decil más rico poseía 14,8\% más de ingresos, de lo que tenían los cuatro deciles más pobres;

La tasa de pobreza y de indigencia, eran de $36,8 \%$ y $13,6 \%$, respectivamente.

En las décadas de los ochenta y noventa, el PIB. per cápita había caído en -14\%. El PIB., per cápita en el año 1995, era menor que en 1980. El analfabetismo en el año 2000, era del 8,4\% de la población mayor de 15 años y por el contrario, el gasto público en educación, era para ese mismo año, del 1,5\% del PIB. (el más bajo de toda América Latina para ese año). La tasa de mortalidad de menores de 5 años para el año 2006, era de 24 por 1000 nacidos vivos. En cuanto al servicio de agua por tubería, para el año 2007, Ecuador tiene uno de los porcentajes más bajos de toda la región ( $73,7 \%$ para el total y $33 \%$ para la población rural). 
Ante esa situación, Ecuador poseía un valor de su presión fiscal del 10,38\%, muy por debajo del promedio para América Latina (13,58\%). Esto ha supuesto una importante limitación respecto al papel redistribuidor del Estado, en las últimas décadas. El gasto social en Ecuador para el año 2004, representaba solo el 6,6\% del PIB., muy por debajo del que poseía en el año noventa (8\%). Era el segundo porcentaje más bajo de toda América Latina, muy por debajo del valor promedio, $15,9 \%$ para el periodo 2004-2005 (Acosta \& Serrano).

La crisis financiera en el Ecuador data de 1999, en el gobierno de Jamil Mahuad y Gustavo Noboa, cuando varios bancos privados de ese país, cerraron o entraron a proceso de rescate financiero, pasando a manos del Estado ecuatoriano. El factor que agravó la crisis, fueron los altos gastos militares efectuados que hizo el Ecuador, para poder afrontar el conflicto bélico del Cenepa de 1995, con el Perú.

El 8 de marzo de 1999, se decretó "El Feriado Bancario" el cual debió ser por 24 horas y terminó después de 5 días. Esto afectó a los bancos, los cuales quebraron y el Estado asumió los costos, los cuales fueron transmitidos a la población a través, de la reducción de gastos sociales y la elevación de los costos de los servicios.

Durante ese mismo año, el Banco Central del Ecuador, con el fin de salvar el déficit presupuestario del Estado que le impedía cubrir con el gasto público, implementó una serie de medidas devaluatorias del Sucre. El efecto de decretar la inflación, fue la depreciación de los ahorros de la población. En respuesta a las medidas del Banco Central, la población ecuatoriana empezó a cambiar masivamente la moneda nacional, Sucre, por el Dólar americano que brindaba más confianza.

En enero de 2000, el gobierno decretó la dolarización a una paridad de 25.000 Sucres por dólar. Por lo cual, quienes tenían ahorros congelados en Sucres, recuperaron sólo una quinta parte; mientras que los que tenían créditos con entidades financieras, vieron reducida su deuda en una quinta parte.

El fin de la crisis bancaria, permitió una tendencia de crecimiento en los créditos. Sin embargo, «hubo que esperar hasta 2004, para que éstos retomasen los niveles históricos antes de la crisis de $1998 »$. 
Como consecuencia del proceso de crisis, quiebra del sistema financiero y dolarización, en el año 2002, los bancos más grandes del país: Pichincha, Produbanco, Guayaquil y Pacífico, mantenían el $63 \%$ de los activos totales de la banca privada y el 59\% de los depósitos, teniendo el Banco Pichincha el $27 \%$ de los activos totales del sistema. Con la recuperación de la economía y la reducción de la tasa de inflación, se produjo el aumento de los depósitos en el sistema financiero, en diciembre de 2002, constituyeron US \$4.331 millones.

Otros cambios considerados de tipo estructural, fue el cambio en los depósitos a la vista, que en 1994 constituían el 50,43\% con respecto a las obligaciones del público, desde el año 2003, fluctuaron entre 68,35\% y alcanzaron el 72,37\% en el año 2010 (Aguilera, 2015).

\section{Resultados}

El sistema financiero de Ecuador es peculiar, debido a que la moneda en la cual está basado es el Dólar de Estados Unidos desde el año 2000. Previamente, existía una moneda nacional como lo fue el Sucre, cuyo tipo de cambio pasó de 5 Sucres por un Dólar en 1927, cuando se fundó el Banco Central del Ecuador (BCE) a 25000 Sucres en enero del 2000 (González \& Romero, 2015).

El sistema bancario ecuatoriano y más concretamente, los intereses económicos-financieros, vinculados a este sector, han demostrado que son capaces de transferir los 5 costos de la crisis financiera al Estado y al resto del país, especialmente a las clases medias y a las masas empobrecidas. Debido a su influencia a nivel del Estado y al hecho de que la casi totalidad de autoridades económicas, ubicadas en los Ministerios de Finanzas, Superintendencia de Bancos, Superintendencia de Compañías, Junta Monetaria y Directorio del Banco Central, han provenido del sector financiero, las políticas del Estado han estado en función de sus intereses.

Esto ha ocasionado, que la posibilidad de solucionar la crisis, no se haga mediante la liquidación de bancos y el pago de las obligaciones contraídas con el patrimonio de los banqueros (tal como lo dispone a la ley) o interviniendo las empresas vinculadas a los grupos financieros, causantes en gran medida de la crisis, sino mediante la asunción de todas las responsabilidades y obligaciones por parte del Estado, siguiendo el ejemplo de la intervención del BCE, a propósito de la crisis del Banco Continental, en 1995. Como lo han señalado reiteradas veces diversos analistas económicos, el peso del salvataje bancario se ha hecho "sin que los banqueros corruptos hayan 
aportado con sus recursos y patrimonio, para financiar las entidades financieras (que), de acuerdo a diferentes estimaciones podría fluctuar entre 2 mil y 5 mil millones de dólares" (Acosta, 2000). El desempeño de cualquier sistema financiero, es el reflejo de la incertidumbre acerca de los prospectos del sector real de una economía. Si los agentes económicos anticipan menor certidumbre, sobre los retornos de su activo debido, por ejemplo, a unas expectativas nada optimistas en el inmediato futuro, debe preverse una desintermediación financiera. En otras palabras, las fluctuaciones económicas, pueden determinar de manera endógena la liquidez y a su vez solvencia del sistema financiero (González \& Romero, 2015).

La crisis del sector financiero ha sido un factor determinante, si bien ésta se ha visto agravada por la inestabilidad política de estos últimos años, la paralización y recesión del aparato productivo y la errática política económica del Estado. En un contexto internacional en el cual una economía como la ecuatoriana es particularmente frágil, la crisis de fin de siglo como la que el país atraviesa, no puede ser explicada, sin embargo, por circunstancias externas. Las causas han sido fundamentalmente internas (Espinosa, 2000).

Durante los últimos casi 15 años, la República del Ecuador, ha logrado alcanzar un cierto nivel de estabilidad económica, a pesar que, en el año 1999, sufriera la peor de sus crisis financieras en toda su historia republicana (Miño, 2008). Parte de esta estabilidad, ha sido apuntalada por un proceso de dolarización que facilitó -en su momento- el control tanto de los procesos hiperinflacionarios, como de devaluación permanente de la moneda de curso legal, hasta aquel entonces: el Sucre (Vera, 2012).

De acuerdo con Eduardo Valencia, quien además presidió la Comisión Investigadora de la Crisis Económica Financiera, creada por este Gobierno el pasado 9 de abril, la crisis arranca con la aprobación en 1994 de la Ley General de Instituciones Financieras. Valencia señala, que las cuantiosas pérdidas ocasionadas se produjeron por decisiones políticas equivocadas del Gobierno de Jamil Mahuad. Valencia afirma, además, que otro motivo fue "la creación de la Ley de la AGD, que no habría podido nacer sino hubiera sido que previamente se aprobó la transitoria No. 42 de la Constitución, que permite al Banco Central otorgar créditos de estabilidad y solvencia a las instituciones financieras, así como crédito para atender el derecho de preferencia de las personas naturales depositantes en las instituciones, que entren en proceso de liquidación". Otras razones para la crisis, según Valencia, se configuran con el feriado bancario, el congelamiento de 
los depósitos y los decretos ejecutivos 685 y 1492, que permite que los Certificados de Depósito Reprogramados (CDRS) vayan a la CFN (La Hora, 2007).

El presidente del Colegio de Economistas de Pichincha, Patricio Almeida, está de acuerdo en que la Ley General de Instituciones del Sistema Financiero, liberó al sistema y se perdieron controles. Afirma que esto "se debía hacer, pero con una Superintendencia de Bancos muy fuerte, que pueda monitorear al sistema financiero. El problema es que se aprobó la Ley y no se pensó en esto y eso permitió, que los bancos otorgaran créditos vinculados, triangulados; financiaran sus empresas que quebraban; financiaran sus grandes negocios y se dieron una vida de rey”. Según Almeida, los créditos de liquidez que entregó el Banco Central, los recuperó casi totalmente y "esas son las ventas que todavía está haciendo el banco: son propiedades entregadas como garantía; el Central está recuperando todos los créditos de liquidez que dio y no es por ahí el problema; el lío está en la emisión de los bonos que hizo el Ministerio de Finanzas y que luego la AGD, entregó indiscriminadamente a los bancos (La Hora, 2007).

\section{Conclusiones}

El sistema financiero ecuatoriano, se vio afectado tras la crisis mundial la cual fue protagonizada por Estados Unidos, repercutiendo en otros países y el mal manejo de sus fondos; para esto intervinieron algunos factores. Uno de los más importantes, fue el cambio de moneda, pasando de Sucre a Dólar, lo cual se produjo después del Feriado Bancario que tuvo partida en el Gobierno de Jamil Mahuad en el año de 1999. A su vez, los precios del petróleo disminuyeron. Como resultado, en 1999, el ingreso por habitante cayó en 9\%, luego de haber declinado el 1\% en 1998 y sólo a partir del año 2000, empieza una leve recuperación que tiende a estancarse en el año 2003.

Los cambios que ha venido sufriendo Ecuador, en base al control de su Sistema Financiero es notable, entendiendo que estabilizarse, tomará algunos años más, requerirá un alto financiamiento y demandará sobre todo un real cambio de orientación del modelo económico. Ya desde hace tiempo, sufría el país dos problemas fundamentales: los riesgos del sistema monetario y la improvisación en la conducción de la política fiscal. Enfrentar lo primero, supone estimular por vías diversas el ingreso de divisas: más exportaciones, más inversión extranjera, más oportunidades de negocios que permitan al Ecuador, acceder a los mercados internacionales y dinamizar la economía nacional. Lo segundo, implica definir una política de gasto por 
prioridades, junto a un esquema de acceso al financiamiento externo, transparente y claro, una simplificación de la política impositiva.

\section{Recomendaciones}

En base al trabajo realizado, la información recolectada y diversas opiniones de expertos en el tema, se puede sugerir las siguientes recomendaciones:

- Hacer un mayor esfuerzo para mantener la estabilidad financiera y fortalecer la competitividad del país.

- $\quad$ Mantener la dolarización.

- Incitar a estrictos controles a administradores del Sistema Financiero.

- $\quad$ El Estado debe diseñar una red de seguridad financiera, que optimice la cantidad de recursos que el sistema financiero mantiene en forma líquida, para enfrentar choques de liquidez, al mismo tiempo, que minimice el riesgo que los problemas de liquidez generen crisis bancarias.

- $\quad$ Se recomienda, además, que el Banco Central del Ecuador, junto con las demás entidades públicas, que tienen entre sus funciones velar por la estabilidad del sistema financiero, diseñe un esquema de red de seguridad financiera a implementarse a la brevedad posible, proceso en el cual es indispensable el diálogo y la búsqueda de la concertación con el sistema financiero privado.

\section{Referencias Bibliográficas}

Acosta,

A.

(2000).

Recuperado

de

http://www.usfq.edu.ec/publicaciones/polemika/Documents/Polemika

001/polemika001_005_articulo001.pdf

Aguilera, Fausto (2015). El Impacto de la crisis financiera y económica internacional en la banca del Ecuador. Quito, Ecuador: Corporación Editorial Nacional.

CEPAL. (2008). Panorama social en América Latina. Recuperado de: https://repositorio.cepal.org/bitstream/handle/11362/1229/1/S0800829_es.pdf

Definición ABC. (s.f.). Recuperado de http://www.definicionabc.com/economia/crisisfinanciera.php

Espinosa, R. (2000). Exigibilidad de los derechos económicos, sociales y culturales. Recuperado de http://www.memoriacrisisbancaria.com/www/articulos/Roque_Espinosa_Crisis_bancaria.pdf 
Gestiopolis. (s.f.). Recuperado de http://www.gestiopolis.com/metodos-y-tecnicas-deinvestigacion/

González, M., \& Romero, P. (12 de Octubre de 2015). La Revista. Recuperado de http://www.larepublica.ec/blog/opinion/2015/10/12/ciclo-economico-sistema-financieroecuatoriano/

Gutiérrez, P. (s.f.). Expansión. Recuperado de http://www.expansion.com/diccionarioeconomico/crisis-financiera.html

La Hora. (24 de Agosto de 2007). La hora nacional. Recuperado de PAÍS: http ://ahora.com.ec/index.php/noticias/show/610147/-1/Pol\%C3\%A9mica_sobre _crisis_econ \%C3\%B3mica_de_1999.html\#.WJdWZBvhDIU

Libertad Digital Economía. (s.f.). Libertad digital economía. Recuperado de http://www.libertaddigital.com/economia/cronologia-de-las-crisis-financieras-del-ultimo-siglo$1276406696 /$

Mendoza, A. (2009). Scribd. Recuperado de http://www.bibliotecapleyades.net/sociopolitica/sociopol_Bigcra sh51.htm

Miño, w. (2008). Breve historia bancaria del Ecuador. Quito, Ecuador: Corporación Editora Nacional.

Pelaéz, E. (Agosto de 2011). Crisis financiera y crisis económica. Recuperado de http://www.ief.es/documentos/recursos/publicaciones/revistas/cuadernos_formacion/12_2011/08 -11.pdf

Pérez, G. (s.f.). Gran depresion.com. Recuperado de Gran Depresion.com: http://www.grandepresion.com/

Shuttleworth, M. (s.f.). Explorable.com. Recuperado de http://explorable.com/es/diseño-de-lainvestigacion-cualitativa

Vera, W. (2012). Incidencia del entorno macroeconómico en el comportamiento de la banca caso Ecuador 1990-2006. Quito, Ecuador: Instituto de los Altos Estudios Nacionales. 